\title{
Polymer-Enforced Crystallization of a Eutectic Binary Hard Sphere Mixture
}

\author{
Anna Kozina ${ }^{a \ddagger}$, Dominik Sagawe $(\dagger)^{a}$, Pedro Díaz-Leyva ${ }^{a, b, \S}$, Eckhard Bartsch ${ }^{a, b, *}$ and Thomas \\ Palberg $^{c}$
}

\section{Received Xth $X X X X X X X X X X 20 X X$, Accepted Xth $X X X X X X X X X 20 X X$}

First published on the web $X$ th $X X X X X X X X X X 200 X$

DOI: 10.1039/b000000x

We prepared a buoyancy matched binary mixture of polydisperse polystyrene microgel spheres of size ratio $\Gamma=$ 0.785 at a volume fraction of $\Phi=0.567$ just below the kinetic glass transition. In line with theoretical expectations, a eutectic phase behaviour was observed, but only a minor fraction of the samples crystallized at all. By adding a short non-adsorbing polymer we enforce interspecies fractionation into coexisting pure component crystals, which in turn also shows signs of intra-species fractionation. We show that in formerly inaccessible regions of the phase diagram binary hard sphere physics are made observable using attractive hard spheres.

Binary colloidal mixtures are valuable model systems for fundamental studies of crystallization $\underline{-1}-\underline{3}$. Detailed predictions of their phase behaviour exist for hard sphere (HS) systems 'of different size ratio $\Gamma=R_{S} / R_{L}$ (where $R_{i}$ are the radii of 'small $(S)$ and large $(L)$ spheres, respectively). These show a sequence of spindle to azeotropic to eutectic phase diagram types with decreasing $\Gamma$, vanishing miscibility in the crystal phase for $\Gamma<0.85$ and a huge variety of crystal structures for compounds $4-9$. For zero miscibility eutectics the inter-species fractionation is expected to cause a pronounced 'slowing of nucleation $\frac{8}{2}$. Polydispersities above $6 \%$ destabilize the crystal phases $\underline{11.12}(10 \%$ in $2 \mathrm{D} \underline{\underline{10}})$, hence additional intra'species fractionation is expected for strongly polydisperse systems ${ }^{13,14}$. Indications of polydispersity altered solidification dynamics have been observed in several HS experiments 15,16 including subtle influences of the skewness of the size distribution $\frac{17}{17}$ but direct evidence of intra-species fractionation is still missing. While many predicted crystal structures have

${ }^{a}$ Institut für Makromolekulare Chemie, Albert-Ludwigs-Universität Freiburg, D-79104 Freiburg, Germany

${ }^{b}$ Institut für Physikalische Chemie, Albert-Ludwigs-Universität Freiburg, D79104 Freiburg, Germany

${ }^{c}$ Institut für Physik, Johannes Gutenberg Universität Mainz, D-55128 Mainz, Germany

$\ddagger$ Current address: Instituto Nacional de Investigaciones Nucleares, $52750 \mathrm{La}$ Marquesa Edo.Mex., Mexico

$\S$ Current address: Departamento de Física, Universidad Autónoma Metropolitana, Iztapalapa, 09340 México, D.F., Mexico by now been observed $\frac{1,2,7,18,19}{}$ and also the general sequence of phase diagram types was confirmed 20 , most parts of the phase diagram escape from a detailed experimental investigation; e.g., the exact locations of phase boundaries in compound forming systems have not yet been determined. Moreover, crystallization kinetics have not been obtained and, except for a recent $2 \mathrm{D}$-study 21 , fractionation into coexisting $L$ and $S$-crystals has not yet been observed. One main reason for this is the interference of crystallization with the glass transition (GT) at large volume fractions 22 . Furthermore, gravity seemingly enhances the trend to dynamically arrest the systems ${ }^{23}$. In addition, it may lead to differential sedimentation and inhomogeneities in composition 24,25 .

In the present paper we avoid sedimentation using a binary mixture of buoyancy matched microgel particles. We further exploit earlier observations on a variety of systems, in which both vitrification can be suppressed ${ }^{26-28}$ and crystallization be accelerated 29 by adding a short chained, non adsorbing polymer. Doing so, one moves from HS to attractive HS (AHS), which in principle may significantly alter both phase behaviour and crystallization kinetics $30-37$. However, using a binary AHS mixture at a size ratio of $\Gamma=0.785$ we here give the first demonstration of the simultaneous precipitation of $S$ - and $L$-crystals over the full range of compositions. Thus, we recover the zero miscibility eutectic solidification process expected for pure HS. Moreover, we provide experimental support for the theoretically expected additional intraspecies fractionation $\frac{38}{3}$ and give a preliminary account of the crystallization kinetics. Further analysis suggests that the final degrees of crystallization as well as the time dependent conversion rates are controlled by both the polydispersity and the polymer-supported ability to form suitable composition fluctuations.

Cross-linked polystyrene $(P S)$ microgel spheres were synthesized by emulsion polymerization, cleaned, dried and resuspended in 2-Ethylnaphtalene $(2 E N)$ as described previously ${ }^{39}$. In this good solvent the particles swell to hydrodynamic radii of $R_{h, S}=(152 \pm 4) \mathrm{nm}$ and $R_{h, L}=(193 \pm 3) \mathrm{nm}$ giving a size ratio of $\Gamma_{h}=0.785$. The hardness of interaction

This journal is $\odot$ The Royal Society of Chemistry [year] Journal Name, 2010, [vol], 1-4 | 1 


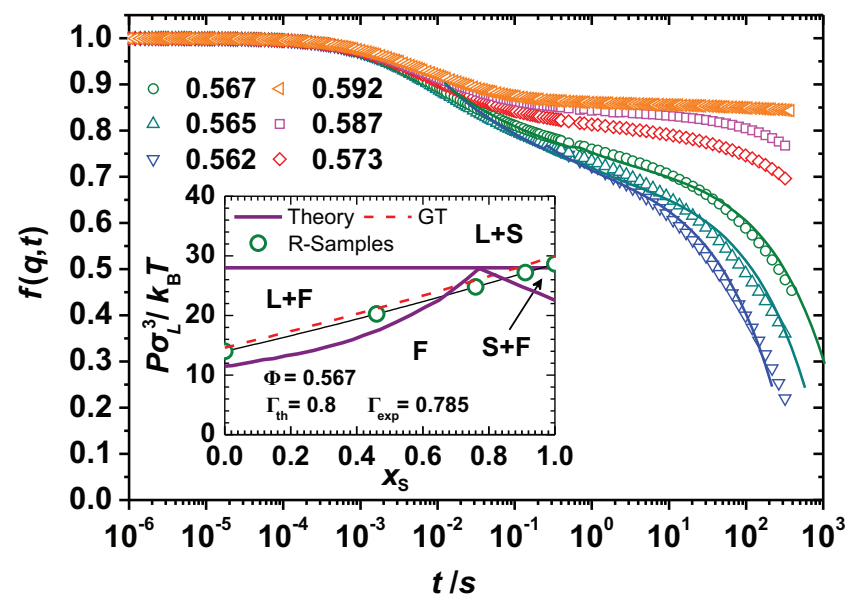

Fig. 1 (colour online) Main: intermediate scattering functions $f(q, t)$ vs. $\log (t)$ for repulsive samples of eutectic composition $\left(x_{S}=0.77\right)$ with increasing $\Phi$ from bottom to top. Inset: binary HS phase diagram in the pressure-composition plane. Thick solid lines: phase boundaries adapted from $\underline{8}$ for $\Gamma_{t h}=0.8$. Thin solid line: osmotic pressure for HS mixtures of experimental size ratio $\Gamma_{h}=0.785$ calculated using the fundamental measure theory equation of state of $\frac{46}{}$. Dashed line: osmotic pressures along the GT-line $(\Phi=0.573)$. Circles: values for $R 1-R 5(\Phi=0.567)$ with partial volume fractions $\phi_{S}=\Phi_{S} / \Phi$ of $0,0.26,0.57,0.83$ and 1 corresponding to molar fractions $x_{S}=\rho_{S} /\left(\rho_{S}+\rho_{L}\right)$ of $0,0.47,0.77,0.92$ and 1 (where $\rho_{i}=\Phi_{i} /(4 \pi / 3) R_{h, i}^{3}$ is the particle number density).

was probed in oscillatory rheological measurements following the procedure described in ${ }^{40}$. Assuming an inverse power potential $U(r) \propto 1 / r^{n}$ we find $n=40 \pm 2^{41}$ which is sufficiently steep to regard the particles as hard spheres. For both pure species the polydispersity index $\sigma=\sqrt{\left\langle R^{2}\right\rangle-\langle R\rangle^{2}} /\langle R\rangle$ is between 0.06 (TEM on dried particles) and 0.08 (form factor measurements). Sedimentation experiments following 42 revealed their expected HS-like phase behaviour. The freezing concentration was then identified with the ideal HS value and the melting points scaled accordingly to obtain $\Phi_{M, S}=0.528$ and $\Phi_{M, L}=0.531$. Further, from the position of the first Bragg reflection of samples at coexistence the lattice spacing and from that effective radii were obtained as $R_{\text {eff }, L}=(170.5 \pm 2)$ $\mathrm{nm}$ and $R_{e f f, S}=(126.2 \pm 3) \mathrm{nm}$ (effective size ratio $\Gamma_{e f f}=$ $0.74)^{43}$. The inset of Fig. 1 shows the expected phase diagram of monodisperse repulsive mixtures. Choosing the eutectic composition and increasing $\Phi$ we monitor the intermediate scattering functions, $f(q, t)$ (Fig. 1 main). A Mode coupling GT is observed for $\Phi_{G T}=0.573 \pm 0.002^{44}$. For $\Gamma \leq 0.8$ the value of $\Phi_{G T}$ hardly varies with composition ${ }^{22}$. The expected GT-line is shown dashed in the inset. Note that neither the eutectic point nor $(L+S)$-coexistence region are accessible.

Three Sample series $(R, E, A)$ were prepared to have $\Phi=$
$0.567 \pm 0.006$, just below $\Phi_{G T}$. $R$-samples to check the repulsive phase behaviour contain no polymer but vary in molar fraction of $S$-particles. $E$-samples of eutectic composition $\left(x_{S}=0.77\right)$ to monitor the effects of attraction contain increasing concentrations, $c_{P}$, of linear $P S\left(R_{g}=13.1 \mathrm{~nm}\right.$, $M_{W}=133 \mathrm{~kg} / \mathrm{mol}$; PSS $\mathrm{GmbH}$, Germany). Strongly attractive $A$-samples of different composition to explore $(L+S)$ coexistence have $c_{P}=12.54 \mathrm{~g} / \mathrm{l}$. Respecting the volume occupied by the colloids, the polymer concentration in the free volume is $c_{P, \text { free }}=43 \mathrm{~g} / \mathrm{l}^{45}$, roughly 1.8 times larger than the overlap concentration $c_{P}^{*}=3 M_{W} /\left(4 \pi N_{A} R_{g}^{3}\right)=23.5 \mathrm{~g} / \mathrm{l}$. (For more details on sample compositions see Fig. 2)

Start of crystallization measurements $(t=0)$ was defined by taking a sample off the homogenizing tumbler and mounting it on a static light scattering goniometer (modified Sofica, SLS Systemtechnik, Germany, $\lambda=405 \mathrm{~nm}, 1 \mathrm{~mW}$ laser illumination, angular range $25^{\circ}-135^{\circ}$, resolution $1^{\circ}$ ). Structure factors, $S(q, t)$, were obtained by time resolved measurements of the scattered intensity, $I(q, t)$, dividing by the appropriate form factors, $P(q)$, obtained from a dilute sample and normalizing by the number density ratio: $S(q, t)=$ $I_{\text {conc }}(q, t) \rho_{\text {dilute }} / P(q) \rho_{\text {conc }}$. Here $q=\left(4 \pi n_{D} / \lambda\right) \sin (\Theta / 2)$ is the scattering vector with $n_{D}$ denoting the suspension refractive index. Zero time structure factors, $S(q, 0)$, only show the meta-stable melt, while at later times $S(q, t)$ is a superposition of melt and crystal contributions. After two months most $S(q, t)$ stopped evolving. The crystal scattering contribution, $S_{C}(q, t)$ was obtained following 47 : $S_{C}(q, t)=S(q, t)-$ $\beta S(q, 0)$. The scaling factor $\beta$ represents the fraction of remaining melt and the total fraction of crystals $X_{C}(t)=1-\beta(t)$. In this approximation we neglect the small changes of melt and crystal composition and thus scattering power with time.

The final $S(q)$ for the $R$-samples in Fig. 2 $(a)$ are dominated by melt scattering. $R 3$ did not crystallize within two years although $\alpha$-relaxation occurred within $10^{3} \mathrm{~s}$. All other samples showed isolated small crystallites. The final crystallinities $X_{C}(\infty) \leq 0.2$ appear to be rather small. Presumably, values given by the lever rule applying to the coexistence regions are further diminished due to polydispersity ${ }^{13}$. In each sample we could isolate individual narrow Bragg-peaks appearing at different $q$. Their average scattering vectors (dotted lines) reasonably well coincide with the melt maxima. Since the calculated osmotic pressures of the mixtures 46 are below the eutectic pressure $P_{E}=28 k_{B} T\left(2 R_{L}\right)^{-3}$ at $\Gamma=0.8^{8}$, our findings are qualitatively consistent with the expected eutectic phase behaviour and with previous results on a $3 \%$ polydisperse mixture with $\Gamma=0.72 \underline{19}$.

The $E$-samples in Fig. $2(b)$ show fluid order up to $c_{P}=$ $9.05 \mathrm{~g} / \mathrm{l}$ and a minimum of the structural relaxation time at $c_{P}=8.10 \mathrm{~g} / \mathrm{l}$. For $c_{P} \geq 10.43 \mathrm{~g} / 1$ we observe crystallization, while for $c_{P} \geq 15.08 \mathrm{~g} / \mathrm{l}$ the samples initially are amorphous again. Their $f(q, t)$ show plateau-like features. $E 9$ and $E 10$ 

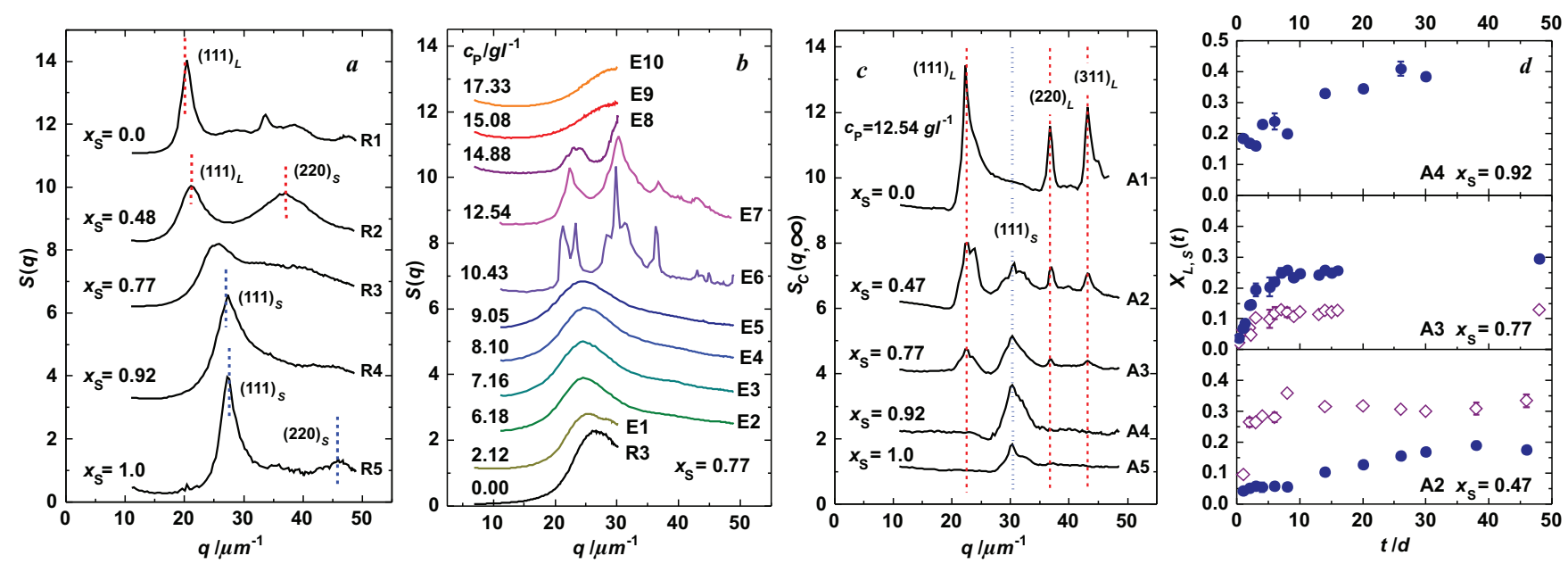

Fig. 2 (colour online) Scattering results. Curves are shifted for clarity. (a) Final structure factors of $R$-samples at different compositions as indicated $(\Phi=0.567)$. Dashed lines indicate location of Miller-indexed reflections averaged over several individual crystallites. $(b)$ Final structure factors for the $E$-samples of $x_{S}=0.77=x_{E}$ and $\Phi=0.567$ with indicated amounts of added polymer. $(c)$ Crystal structure factors of $A$-samples with location of Miller-indexed reflections of the pure samples averaged over several individual crystallites (dashed lines) (d) Temporal development of the fraction of crystals for the three mixtures $A 4-A 2$. Filled symbols denote $S$-crystals, open symbols denote $L$-crystals.

finally started crystallizing after two years. We then varied the composition with $c_{P}$ above the crystallization threshold. Fig. 2( $c)$ shows the $S_{C}(q, \infty)$ of the $A$-samples with $c_{P}=12.54$ $\mathrm{g} / \mathrm{l}$. Large numbers of small crystallites appeared within days and grew to final sizes $(0.4-0.8 \mathrm{~mm})$ within several weeks. Absence of additional peaks and shifts in peak positions with $x_{s}$ excludes the formation of compounds and of substitutional crystals. By comparison to the $S_{C}(q, \infty)$ of the two pure samples we identify $A 2$ and $A 3$ to show crystals of both species, while $A 4$ contains $S$-crystals only. This simultaneous occurrence of both crystal species shows that the systems are above the eutectic pressure (c.f. Fig. 11). Within experimental uncertainty the observed lattice constants and thus the particle number densities $n$ equal those of the pure samples. Using the latter and the effective radii, $R_{e f f, i}$, we obtain the volume fractions for both coexisting crystal species to be $\Phi=0.73 \pm 0.02$ for all samples. This is remarkably close to the maximum packing fraction obtainable in a close packed structure. At this size ratio similar compressions have so far only been reached, when additional gravitational compression of sedimented crystals occurred 36 .

From time resolved measurements the fraction of crystalline material, $X(t)$, was obtained by integrating the intensity in the corresponding peak regions: $X_{L, S}(t)=c \int d q S_{C}(q)$, choosing $c$ such that $X_{L}(t)+X_{S}(t)=X_{C}(t)=1-\beta(t)$. Fig. $2(d)$ shows that at $x_{S}=0.92$ about $20 \%$ of the $S$-particles form $S$-crystals already at early times which grow for about a month; at $x_{S}=0.77,10 \%$ of each species form crystals simultaneously; the final crystallinity is reached after 10 days; for $x_{S}=0.47$ starting again from about $10 \%$ of each species, $S$-crystal conversion accelerates after $L$-crystals have nearly reached their final crystallinity. In all samples the degree of crystallization $X_{C}(\infty) \approx 0.4-0.6$ is considerably enhanced over that reached in the $R$-samples. Moreover, at this $c_{P}$ the final $X_{S}$ of $0.42,0.4,0.3$ and 0.2 roughly scale with the number fractions of 1.0, 0.92, 0.77 and 0.47, showing that about $40 \%$ of the $S$-particles are able to solidify via intra-species fractionation. Similarly, for the $L$-particles we find $X_{L}$ to be $0.13,0.34$ and 0.6 for $\left(1-x_{S}\right)$ being $0.23,0.53$ and 1.0, i.e. more than half of the particles crystallize.

Summarizing, we find the theoretically expected eutectic phase behaviour for a purely repulsive HS mixture of $\Gamma=0.8$ and $\Phi=0.567$ i.e. fluid-solid coexistence or pure fluid ${ }^{4}-\underline{8}$, but access to both the eutectic point and the $(L+S)$-coexistence region is blocked by a kinetic glass transition. Upon addition of non adsorbing polymer, however, the eutectic point in terms of polymer concentration can be located at $9.05 \mathrm{~g} / \mathrm{l}$ $<c_{P, E} \leq 10.43 \mathrm{~g} / \mathrm{l}$. As the pressure, $P$, surmounts the eutectic pressure $P_{E}$, i.e. the freezing pressure, $P_{F}\left(x_{E}\right)$, measured at the eutectic composition, $(L+S)$-coexistence is observed for all compositions. Recent work on one component AHS 36.37 showed that both $P(\Phi)$ and $P_{F}$ shift to lower values upon increasing the attraction. Our work suggests that for binary AHS $P_{F}$ drops faster than $P(\Phi=0.567)$. However, theoretical predictions for composition dependent equations of state and the phase diagram of binary AHS are eagerly awaited to quantitatively rationalize our observations in a plot analogous to Fig. 
Being above $P_{E}$, our mixtures should convert completely to $S$ - or $L$-crystals. Instead, we find a pronounced amorphous background signal and broad pyramid-shaped peaks composed from a superposition of a large number of individual Bragg reflections located at slightly different $q$. In addition to this overall incomplete conversion we observe an absence of $L$-crystals at large $x_{s}$ (already seen in ${ }^{19,25}$ ), an increased initial formation of $S$-crystals in $A 4$, their delayed formation in $A 2$. This supports theoretical expectations that conversion proceeds via combined inter $-\frac{8}{-}$ and intra-species fractionation 38 . In both cases the conversion efficiency should be correlated with the possibility to form combined density and composition fluctuations. The addition of polymer generally enhances the dynamics of such fluctuations 28 as long as the system remains un-trapped by the attractive glass. However, conversion efficiency also relies on the availability of similar sized partner particles to form a stable fluctuation. Their concentration in the remaining melt changes in time. Majority species and component particles crystalize first. The enrichment of the minority particles changes the corresponding fluctuation spectra and allows further conversion. However, particles from the wings of a size distribution or of a very diluted species simply stay too dispersed to crystallize.

In this study we have recovered some interesting aspects of HS-crystallization in an AHS system. In future, the controlled use of depletion attraction in formerly inaccessible regions of the phase diagram may allow detailed and quantitative studies of the phase behaviour at other size ratios, kinetics of compound formation or other interesting problems of condensed matter physics.

We thank H. Moschallski for particle synthesis and purification. Financial support of the DFG (Pa459/8, 12-14, 16; Ba1619/2; SFB 428; SFB TR6), the EU (CT-2003-504 712), and the Graduate School of Excellence, Material Science in Mainz, is gratefully acknowledged.

\section{References}

1 P. Bartlett and W. van Megen in: A. Mehta (Ed.), Granular Matter (Springer, New York, 1994).

2 U. Gasser, J. Phys.: Condens. Matter, 21, 203101 (2009).

3 N. J. Lorenz et al., J. Phys.: Condens. Matter, 21, 464116 (2009).

4 P. Bartlett, J. Phys.: Condens. Matter, 2, 4979 (1990).

5 W. G. T. Kranendonk and D. Frenkel, Mol. Phys., 72, 679 (1991).

6 X. Cottin and P. A. Monson, J. Chem. Phys., 99, 8914 (1993) and ibid. 102, 3354 (1995).

7 M. D. Eldridge et al., Mol. Phys., 84, 395 (1995).

8 S. Punnathannam and P. A. Monson, J. Chem. Phys., 125, 024508 (2006).

9 L. Filion and M. Dijkstra, Phys,. Rev. E, 79, 064714 (2009).
10 S. Pronk and D. Frenkel, Phys. Rev. E, 69, 066123 (2004).

11 P. G. Bolhuis and D. A. Kofke, Phys. Rev. E, 54, 634 (1994).

12 D. A. Kofke and P. G. Bolhuis, Phys. Rev. E, 59, 618 (1999).

13 H. Xu and M. Baus, J. Phys. Chem., 118, 5045 (2003).

14 M. Fasolo and P. Sollich, Phys. Rev. Lett., 91, 068301 (2003) and Phys. Rev. E, 70, 041410 (2004).

15 H. J. Schöpe, G. Bryant and W. van Megen, Phys. Rev. E, 74, 060401 (2006) and J. Phys. Chem., 127, 084505 (2007).

16 S. Iacopini, T. Palberg and H. J. Schöpe, J. Chem Phys., 130, 084502 (2009).

17 S. Martin, G. Bryant and W. van Megen, Phys. Rev. E, 67, 061405 (2003) and ibid. 71, 021404 (2005).

18 P. Bartlett, R. H. Ottewill and P. N. Pusey, J. Chem. Phys., 93, 1299 (1990).

19 W. J. Hunt, R. Jardine and P. Bartlett, Phys. Rev. E, 62, 900 (2000).

20 A. Meller and J. Stavans, Phys. Rev. Lett., 68, 3646 (1992).

21 N. Geerts, S. Jahn and E. Eiser, J. Phys.: Condens. Matter, 22, 104111 (2010).

22 W. Götze and T. Voigtmann, Phys. Rev. E, 67, 021502 (2003).

23 J. Zhu et al., Nature, 387, 883 (1997).

24 N. Lorenz, H. J. Schöpe and T. Palberg, J. Chem. Phys., 131, 134501 (2009).

25 M. Leocmach, C. P. Royall and H. Tanaka, Europhys. Lett., 89, 38006 (2010).

26 K. N. Pham et al., Science, 296, 104 (2002).

27 S.-H. Chen, W.-R. Chen and F. Mallamace, Science, 300, 619 (2003).

28 T. Eckert and E. Bartsch, Phys. Rev. Lett., 89, 125701 (2002).

29 C. Smits et al., Phase Transitions, 21, 157 (1990).

30 A. P. Gast, C. K. Hall and W. B. Russel, J. Colloid Interf. Sci., 96, 251 (1984).

31 H. N. W. Lekkerkerker et al., Europhys. Lett., 20, 559 (1992).

32 W. Poon, F. Renth and R. M. L. Evans, J. Phys. Condens. Matter, 12, A269 (2000).

33 W. C. K. Poon, J. Phys.: Condens. Matter, 14, R859 (2002).

34 M. S. Romero-Cano and A. M. Puertas, Soft Matter, 4, 1242 (2008).

35 V. J. Anderson and H. N. W. Lekkerkerker, Nature, 416, 811 (2002).

36 S. Buzzaccaro, R. Rusconi and R. Piazza, Phys. Rev. Lett., 99, 098301 (2007).

37 T. Zykova-Timan, J. Horbach and K. Binder, J. Chem. Phys., 133, 014705 (2010).

38 M. Fasolo and P. Sollich, J. Chem. Phys., 122, 07904 (2005).

39 E. Bartsch et al., J. Non-Cryst. Solids, 307-310, 802 (2002).

40 H. Senff and W. Richtering, J. Chem. Phys., 111, 1705 (1999).

41 A. Kozina, Ph.D. thesis, Freiburg, 2009.

42 S. E. Paulin and B. J. Ackerson, Phys. Rev. Lett., 64, 2663 (1990).

43 The difference of particles sizes from dynamic and static scattering experiments is characteristic of microgel particles and can be traced back to the different visibilities of dangling chain ends at the particle surfaces; see E. Bartsch et al., Ber. Bunsenges. Phys. Chem., 102, 1597 (1998).

44 Determined by applying the asymptotic power law of mode coupling theory (MCT) for the structural or $\alpha$-relaxation time; see W. Götze and L. Sjögren, Rep. Prog. Phys., 55, 241 (1992).

45 See S. M. Illet et al., Phys. Rev. E, 51, 1344 (1995) for the conversion procedure.

46 R. Roth, J. Phys.: Condens. Matter, 22, 063102 (2010).

47 J. L. Harland and W. van Megen, Phys. Rev. E, 55, 3054 (1997). 